\title{
OPEN Organic light-emitting devices based on conducting polymer treated with benzoic acid
}

\begin{abstract}
Hwa Seung Kang ${ }^{1,3}$, Dae Hun $\mathrm{Kim}^{2,3}$ \& Tae Whan Kim ${ }^{1,2} \bowtie$
We report on the enhanced conductivity of the benzoic-acid-treated poly(3,4-ethlenedioxythiophe ne):poly(styrene sulfonate) (PEDOT:PSS) electrode for use in highly flexible, organic light-emitting devices (OLEDs). The conductivity of the benzoic-acid-treated PEDOT:PSS electrode increased from 1 to $1583.2 \mathrm{~S} / \mathrm{cm}$, in comparison with that of the pristine PEDOT:PSS electrode, due to a complex factor of the $\mathrm{H}^{+}$mole $\%$ and the dielectric constant of the benzoic solution. Among the post-treatment methods of the PEDOT:PSS electrodes, the operating voltage at $1000 \mathrm{~cd} / \mathrm{m}^{2}$ of OLEDs fabricated utilizing the PEDOT:PSS electrode with the benzoic acid treatment has the lowest value, and its maximum luminance is $24,400 \mathrm{~cd} / \mathrm{m}^{2}$, which are 1.54 and 2.15 times higher than those of OLEDs using PEDOT:PSS electrodes treated with dimethyl sulfoxide and methanol, respectively. The luminance of a flexible OLED with a benzoic-acid-treated PEDOT:PSS electrode after 1400 bending cycles decreased to $83 \%$ of the initial luminance, resulting in excellent mechanical stability.
\end{abstract}

In recent years, the flexible organic light-emitting devices (OLEDs) have received attention as excellent candidates in their potential applications in next-generation wearable devices and multifunctional intelligent systems. In particular, researches on the flexible transparent electrodes have been conducted due to the performance enhancement of the flexible OLEDs. Most transparent electrode for OLEDs have used the indium tin oxide (ITO) due to its excellent properties such as high transparency and high conductivity ${ }^{1,2}$. However, ITO electrode is not suitable as a flexible transparent electrode due to its inherent brittleness ${ }^{3}$. Alternative transparent electrodes such as metallic wires ${ }^{4-8}$, carbon materials ${ }^{1,9-13}$, and conducting polymers ${ }^{14-18}$ have been studied to replace the ITO electrode. Poly (3,4-ethylenedioxythiophene):poly (styrene sulfonate) (PEDOT:PSS) has been widely utilized as a transparent electrode material owing to their outstanding properties of high optical transparency in the visible range, high mechanical stability, and high electrical conductivity ${ }^{19-22}$. However, since the pristine PEDOT:PSS electrode has a very low conductivity compared to other alternative flexible transparent electrodes ${ }^{23,24}$, the performance of OLEDs with PEDOT:PSS electrode is significantly reduced. Therefore, studies to increase conductivity of PEDOT:PSS electrode for high efficient flexible OLEDs is required.

Recently, many studies have been conducted to increase the conductively of PEDOT:PSS electrode. The post treatment of PEDOT:PSS electrode including organic solvent ${ }^{25,26}$, zwitterions ${ }^{27}$, salt ${ }^{28}$ or acid ${ }^{29-31}$ have been reported as alternative to enhance conductivity. The enhancement of conductivity of PEDOT:PSS electrode with the post treatment originate from the reduction of columbic interaction due to phase separation between PEDOT and PSS. Among the various materials, the conductivity of the sulfuric acid treated PEDOT:PSS electrode was reported to be increased more than $3200 \mathrm{~S} / \mathrm{cm}^{32}$. However, the sulfuric acid, a strong acid that can damage the flexible devices, is not suitable for use as materials to enhance conductivity of PEDOT:PSS electrode ${ }^{33}$. To reduce damage to the flexible device, conducting weak acid treatment on the PEDOT:PSS electrode results in lower conductivity than the strong acid treatment. Therefore, it is necessary to consider mechanisms and process methods that can further improve conductivity of PEDOT:PSS electrode though the weak acid treatment.

In this study, we report mechanism to improve conductivity of PEDOT:PSS electrode with the weak acid treatment for the high efficient OLEDs. The conductivity of PEDOT:PSS with the benzoic acid treatment is enhanced more than $1500 \mathrm{~S} / \mathrm{cm}$, and its conductivity enhancement is related to the removal of the insulator PSS in the PEDOT:PSS film due to the hydrogen ion in the benzoic acid solution. In the more detail, the conductivity of PEDOT:PSS with the benzoic acid treatment improves as the variation of parameters such as $\mathrm{PH}$ values and dielectric constant. The external quantum efficiency of the flexible OLEDs with the weak acid treatment has almost the same external quantum efficiency as the OLEDs using the ITO electrode.

\footnotetext{
${ }^{1}$ Department of Information Display Engineering, Hanyang University, Seoul 04763, Korea. ${ }^{2}$ Department of Electronics and Computer Engineering, Hanyang University, Seoul 04763, Korea. ${ }^{3}$ These authors contributed equally: Hwa Seung Kang and Dae Hun Kim. ${ }^{\square}$ email: twk@hanyang.ac.kr
} 


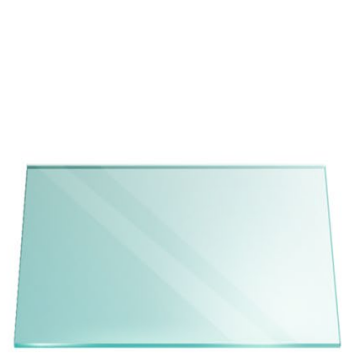

Glass Substrate

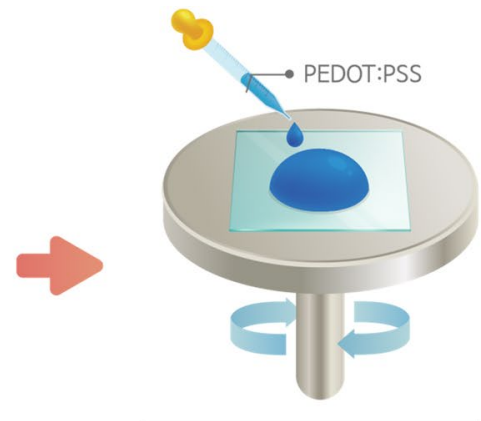

Spin-coating

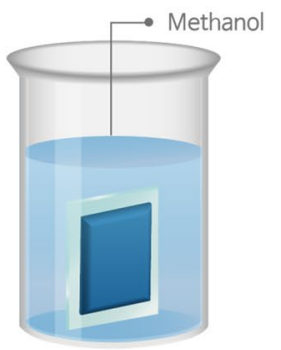

Rinse for $5 \mathrm{~min}$

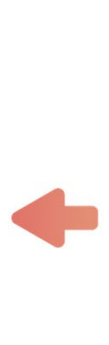

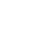

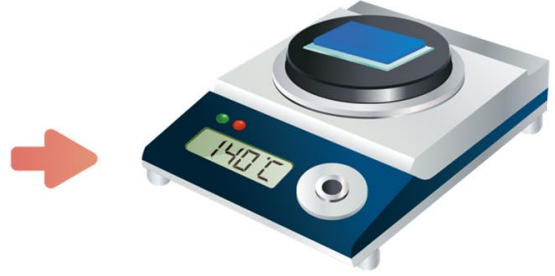

Annealing for $20 \mathrm{~min}$
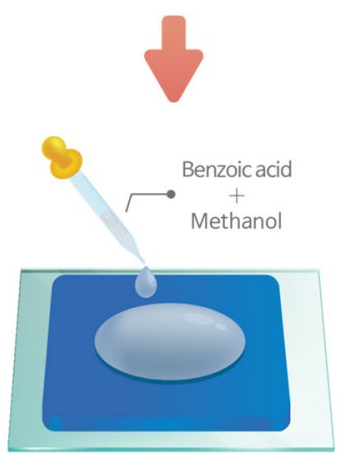

Drop casting for $5 \mathrm{~min}$

Annealing for $20 \mathrm{~min}$ Figure 1. Schematic diagrams of fabrication process for the PEDOT:PSS electrode with benzoic acid treatment
(Kang et al.).

\section{Methods}

The glass substrates were cleaned in acetone, methanol and de-ionized water with ultra-sonication for $10 \mathrm{~min}$, and the cleaned glass substrates were treated by the ultraviolet/ozone lamp for $20 \mathrm{~min}$. A schematic diagram of fabrication process for the PEDOT:PSS with the benzoic treatment was shown in Fig. 1. After the PEDOT:PSS solution was deposed on the cleaned glass substrate by spin-coating at $3000 \mathrm{rpm}$ for $60 \mathrm{~s}$, the PEDOT:PSS film was annealed at $140{ }^{\circ} \mathrm{C}$ on a hot plate for $20 \mathrm{~min}$. Then, the benzoic acid powder was mixed a methanol, and then the benzoic acid solution was drop-casting on the PEDOT:PSS film on the hot plate for 5 min at $140{ }^{\circ} \mathrm{C}$. The benzoic acid treated PEDOT:PSS film was rinsed to remove the residual benzoic acid. After the sample had been transferred into an evaporation chamber, the organic layers and the $\mathrm{LiF} / \mathrm{Al}$ electrodes were deposited at $25^{\circ} \mathrm{C}$ and a system pressure of $1.2 \times 10^{-6}$ Torr. The deposition rates of the organic layers and the metal layer were approximately 1.0 and $2.0 \AA / \mathrm{s}$, respectively.

The sheet resistance was measured by using a sheet resistance meter (FPP-40 K, DASOL ENG). The absorption spectra and the transmittance measurements were performed by using an ultraviolet (UV)-visible spectrophotometer (Lambda 650S, Perkin Elmer). The dielectric constant was measured by liquid dielectric constant meter (M871, Nihon Rufuto). The PH is measured by the surface chemical compositions changes were acquired by using X-ray photoelectron spectroscopy (XPS) (K-Alpha + XPS, Thermo Fisher Scientific). The surface roughness, the phase and film thickness of the film were obtained by using atomic force microscopy (AFM) (XE-100, Park Systems). The current density-voltage-luminance characteristics were measured by using a programmable source meter with built-in current and voltage measurement units (M6100, McScience) and a Minolta CS1000 camera. All OLEDs electrical characteristics were measured at the atmosphere conditions.

\section{Results and discussion}

Figure 2a shows the sheet resistances and the conductivities of the PEDOT:PSS films treated with (A) dimethyl sulfoxide (DMSO) or methanol containing (B) 0, (C) 10, (D) 30, (E) 50, and (F) 70 wt.\% benzoic acid. The sheet resistances of the PEDOT:PSS films treated with solutions (A) and (B) are 245.5 or $266.1 \Omega / \mathrm{sq}$, and the corresponding conductivities are 909.1 or $714.2 \mathrm{~S} / \mathrm{cm}$, respectively. Thus, the corresponding conductivities of the PEDOT:PSS treated with solutions (A) and (B) are improved in comparison with that of the pristine PEDOT:PSS with a conductivity below $1 \mathrm{~S} / \mathrm{cm}^{34}$. The solutions of the methanol and DMSO have the high dielectric constant. The high dielectric constant of solutions weakens the Columbic interaction between PEDOT and PSS, resulting in an increase of the conductivity of PEDOT:PSS due to the formation of the large PEDOT domain in PEDOT:PSS ${ }^{23,25}$. The sheet resistances of the PEDOT:PSS treated with solutions (C), (D), (E), and (F) are 220.3, $190.4,162.4$ and $199.4 \Omega / s q$, and the corresponding conductivities are 1010.1, 1234.6, 1583.2 and $1114.8 \mathrm{~S} / \mathrm{cm}$, respectively. The conductivities of PEDOT:PSS with benzoic acid treatment are enhanced in comparison with the 
(a)

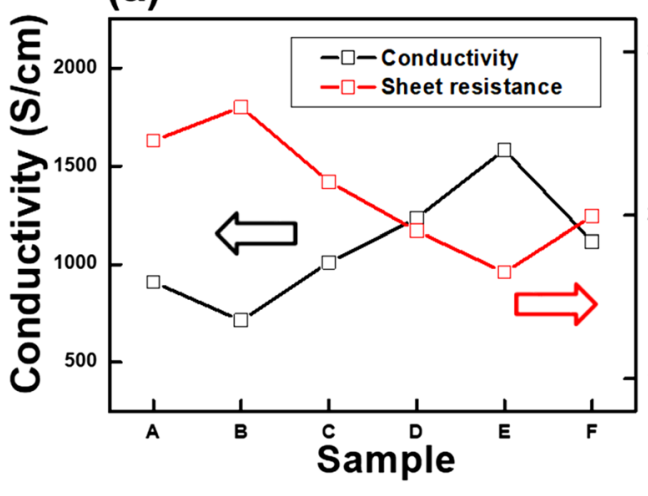

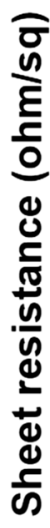

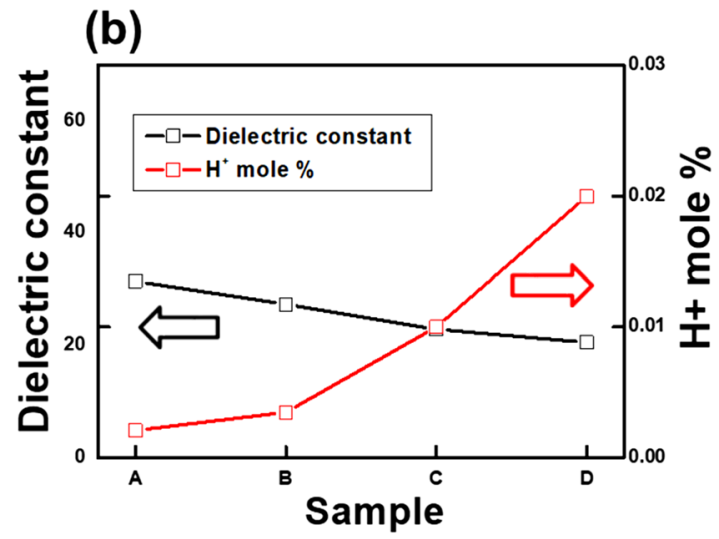

Figure 2. (a) Sheet resistances and conductivities of the PEDOT:PSS films with DMSO, methanol and benzoic acid treatments, and (b) the dielectric constants and the $\mathrm{H}+$ mole $\%$ of benzoic acid solution with various weight concentration of the benzoic acid (Kang et al.).

those of the different organic solvent treatments. The conductivity of PEDOT:PSS with benzoic acid concentration of $50 \%$ has highest value in that of the various benzoic acid concentration. For a detailed analysis of the conductivity improvement of the PEDOT:PSS electrode with benzoic acid treatment, the dielectric constants and the $\mathrm{H}^{+}$mole \% of benzoic acid solution are measured, as shown in Fig. $2 \mathrm{~b}$. The $\mathrm{H}^{+}$mole \% of benzoic acid solutions with benzoic acid concentrations of (A) 10 , (B) 30 , (C) 50 , and (D) $70 \mathrm{wt} \%$ are $2.1 \times 10^{-3}, 3.5 \times 10^{-3}, 1.0 \times 10^{-2}$, and $2.0 \times 10^{-2}$, respectively, and the dielectric constants of the those solutions with benzoic acid concentrations of (A), (B), (C), and (D) are 31.5, 27.4, 22.9, and 20.6, respectively. The $\mathrm{H}^{+}$mole $\%$ of benzoic acid solution gradually increases as the benzoic acid concentration increases. The increases of $\mathrm{H}^{+}$mole $\%$ is related to the ionization of benzoic acid solution. Alcohols are amphiprotic substance, much like water, which can act as both an acid and a base by donating or accepting a proton. According to the Brønsted-Lowry definition, methanol can act as a Brønsted base, which can accept a proton ${ }^{35}$. Therefore, the proton can transfer from benzoic acid to methanol, resulting in the formation of the hydrogen ions in the benzoic acid solution. The hydrogen ions in the benzoic acid solution interact the negatively charged PSS, resulting in the formation of the neutral charge PSSH. The Columbic interaction between conductive PEDOT and insulator PSS is reduced, and the electrical conductivity of PEDOT:PSS is enhanced by phase separation between conductive PEDOT and insulator PSS. The dielectric constant is another factor for the enhancement of conductivity, which reduces the Columbic interaction between conductive PEDOT and insulator PSS ${ }^{36,37}$. As a result, the conductivity of the PEDOT:PSS with the benzoic acid treatment increases due to a complex factor of the $\mathrm{H}^{+}$mole $\%$ and the dielectric constant of the benzoic solution. Even though the $\mathrm{H}^{+}$mole \% and the dielectric constant of benzoic acid at the concentration of 70 wt.\% is the highest, it can be seen that the conductivity of the PEDOT:PSS with benzoic acid treatment is lowered due to the residual benzoic acid. The low conductivity benzoic acid residues reduce the conductivity of the PEDOT:PSS.

Figure 3 shows the schematic illustrations of the conductivity enhancement mechanism of PEDOT:PSS in detail. The pristine PEDOT:PSS has core-shell structure composed of ionic bond between the conductive PEDOT and the insulating PSS, and generally contains gel like particles with an insulating PSS shell to stabilize the conductive PEDOT rich particles. The hydrophilic PSS chain is at the outer shell and the hydrophobic PEDOT segments are in the core, which is formed by columbic repulsion among the PSS chains ${ }^{38,39}$. The hydrophilic PSS shell can be used to increase the water solubility of PEDOT:PSS, but the conductivity of PEDOT is reduced because of the impact of the insulting PSS shell. However, after methanol is added to PEDOT:PSS, the methanol, which has a high dielectric constant as a polar solvent, causes a screen effect between the positively charged PEDOT and the negatively charged PSS. When the methanol penetrated into the PEDOT:PSS film, the PSS shell is separated from the PEDOT core due to decrease of the coulomb attraction between the positively charged $\mathrm{PEDOT}^{+}$and the negatively charged PSS $^{-}$, resulting in the formation of the PEDOT aggregation. The increases of conductive PEDOT aggregation means the enhancement of the conductivity of PEDOT:PSS. In the benzoic acid treatment, after the negatively charged PSS ${ }^{-}$is converted the neutral PSSH due to the hydrogen bonding between PSS $^{-}$of PEDOT:PSS and the $\mathrm{H}^{+}$ion of benzoic acid solution, the PSSH chains are rinsed away from the PEDOT core, resulting in the reconstruction of shape of an extended PEDOT coil. When the hydrogen bonding effect between $\mathrm{H}^{+}$of benzoic acid and PSS ${ }^{-}$of PEDOT:PSS is added to the screen effect of the methanol solvent, the region of PEDOT aggregation is increased than that of the methanol solvent treated PEDOT aggregation, which leads to an improvement in conductivity of PEDOT:PSS.

The transmittance spectra of PEDOT:PSS films and ITO film in the visible region are shown in Fig. $4 \mathrm{a}$. The intensities of transmittance spectra for the pristine PEDOT:PSS, and the PEDOT:PSS with methanol, benzoic acid, and DMSO treatments have almost the same values in the visible range between 400 and $800 \mathrm{~nm}$. Moreover, the intensity of transmittance spectra for the PEDOT:PSS with benzoic acid treatment is higher than that for the ITO between the $400 \mathrm{~nm}$ and $550 \mathrm{~nm}$. The transmittance of the PEDOT:PSS with benzoic acid treatment have high values from $89.59 \%$ to $85.17 \%$, which can be used transparent electrodes. Bending properties of the PEDOT:PSS electrode with benzoic acid treatment on poly(ethylene 2,6-naptalate) (PEN) substrate are shown in Fig. 4b. The sheet resistance is increased 1.18 times higher than initial resistance after 1300 cycles of bending 
Pristine PEDOT:PSS
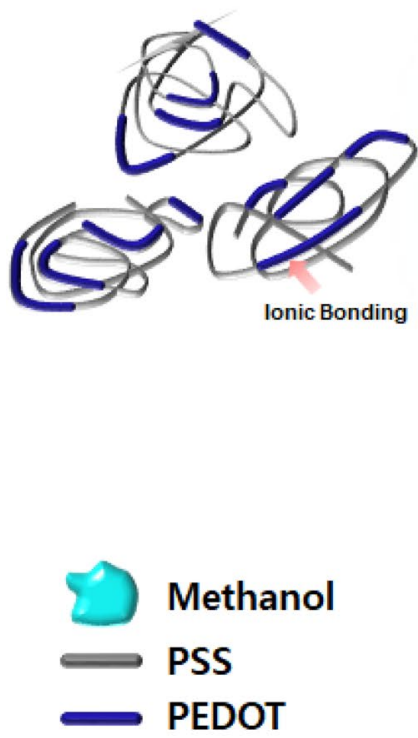

Hydrogen ion
Methanol treatment

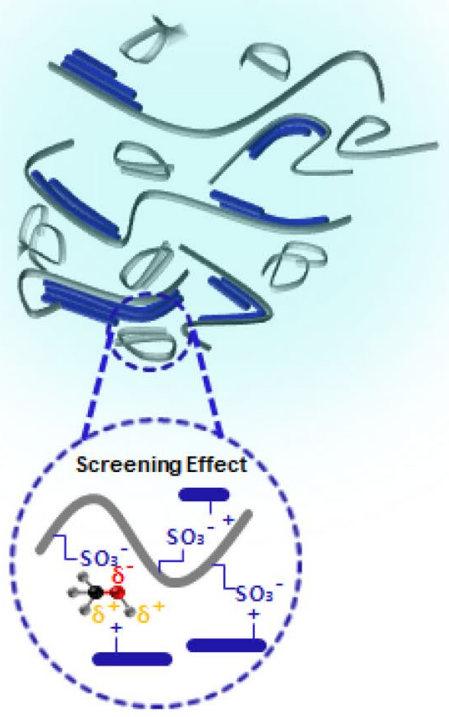

Small PEDOT Aggregation
Benzoic acid treatment

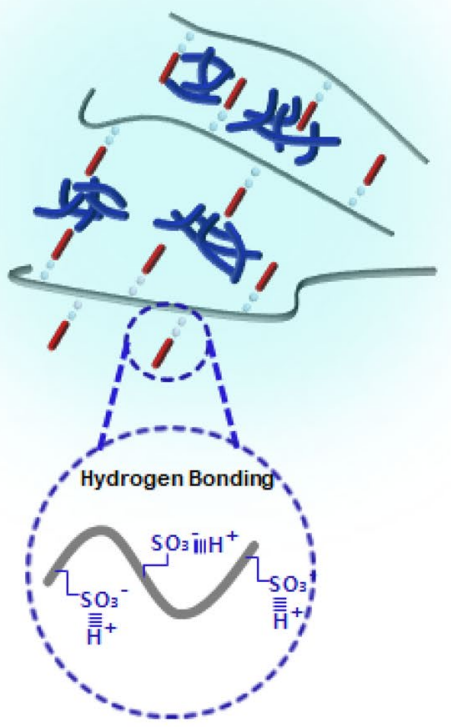

Large PEDOT Aggregation

Figure 3. Schematic illustrations of the conductivity enhancement mechanism of PEDOT:PSS film with benzoic acid treatment (Kang et al.).

(a)

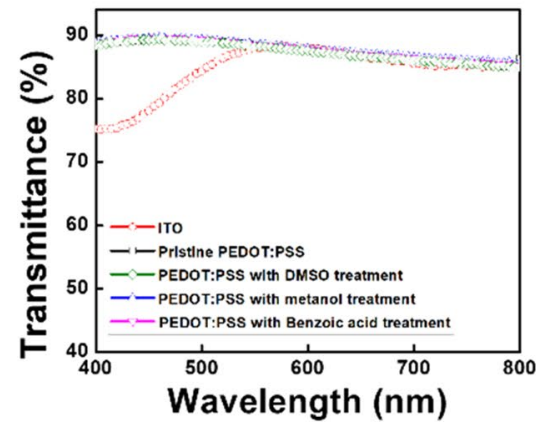

(d)

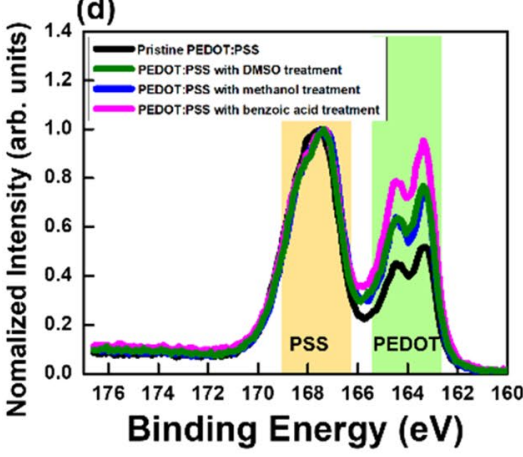

(b)

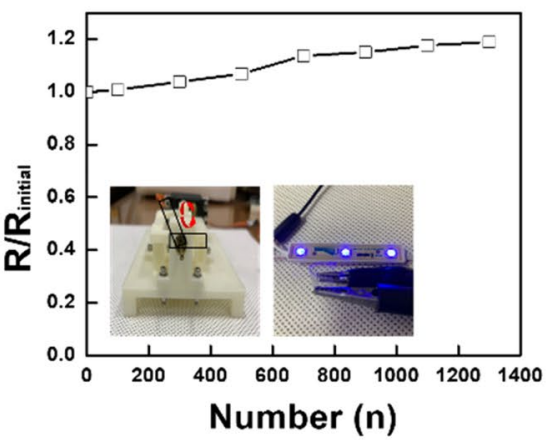

(e)

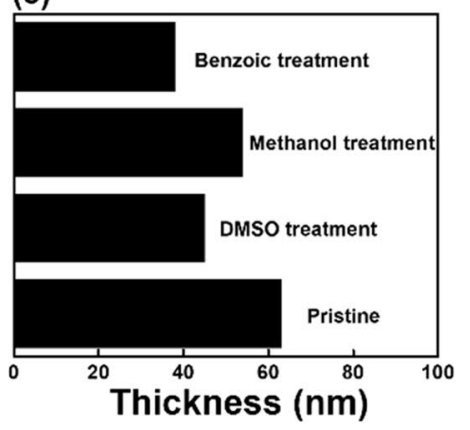

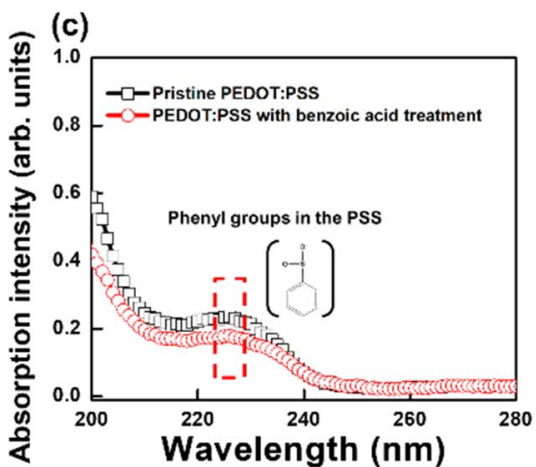

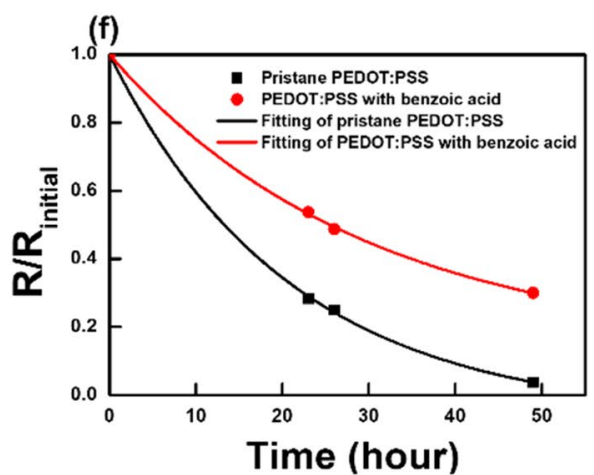

Figure 4. (a) Transmittance spectra of the ITO, the pristine PEDOT:PSS, and the PEDOT:PSS with DMSO, methanol, and benzoic acid treatments. (b) Bending stability for the PEDOT:PSS electrode with benzoic acid treatment on a PEN substrate. (c) Ultra-visible absorption spectra of the PEDOT:PSS films before and after benzoic acid treatment. (d) S2p XPS spectra of pristine PEDOT:PSS, and PEDOT:PSS with DMSO, methanol, benzoic acid treatments. (e) Film thickness of pristine PEDOT:PSS, and PEDOT:PSS with DMSO, methanol, and benzoic acid treatments. (f) Variations in the ratios of the sheet resistances to the initial sheet resistances for the pristine PEDOT:PSS and the PEDOT:PSS with benzoic acid treatment for an $80 \%$ humidity (Kang et al.). 
(a)

\section{Pristine PEDOT:PSS}

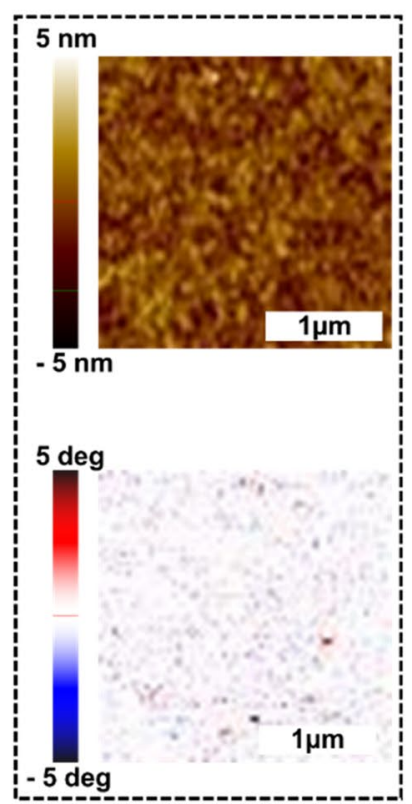

(b)

\section{Methanol treatment}

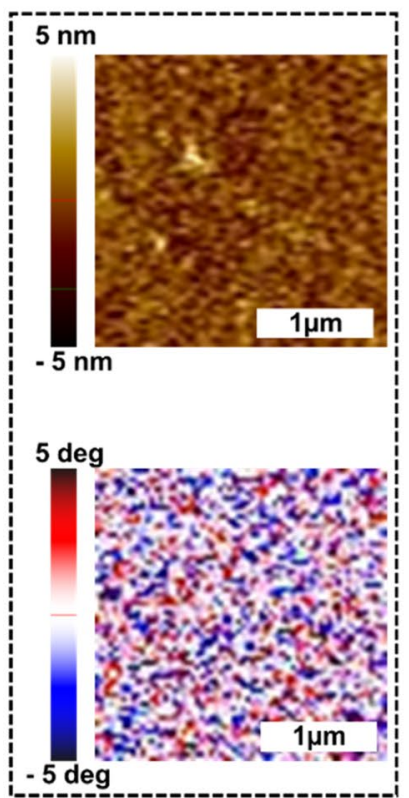

(c)

\section{DMSO treatment}

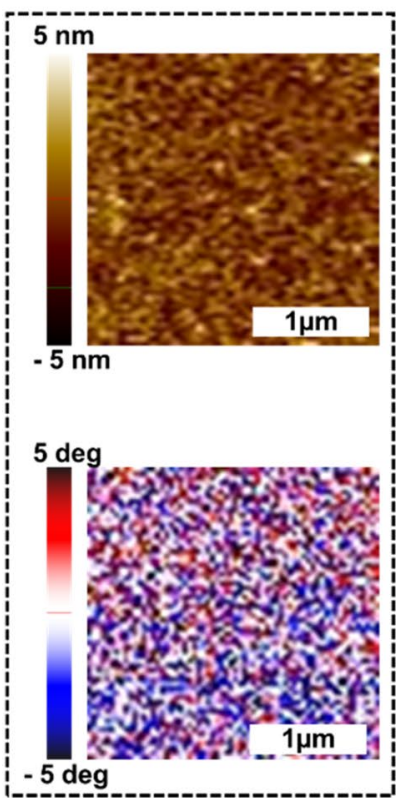

(d)

Benzoic acid treatment

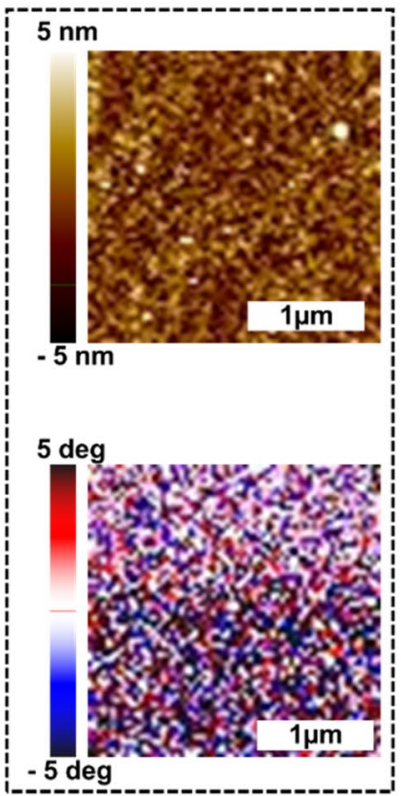

Figure 5. Atomic force microscope topography images of (a) pristine PEDOT:PSS, and PEDOT:PSS with (b) methanol, (c) DMSO, and (d) benzoic acid treatments, and phase images of (e) pristine PEDOT:PSS, and PEDOT:PSS with (f) methanol, (g) DMSO, and (h) benzoic acid treatments (Kang et al.).

at a $5 \mathrm{~mm}$ of bending radius. This result shows the excellent mechanical stability of the PEDOT:PSS electrode with benzoic acid treatment after bending process. Figure 4c shows absorption spectra of the PEDOT:PSS films with and without benzoic acid treatment. The absorption peak of PEDOT:PSS film at $225 \mathrm{~nm}$ originates from substituted phenyl groups in PSS ${ }^{40}$. The reduction of absorption intensity of PEDOT:PSS after the benzoic acid treatment indicates that the decreases in the amount of PSS from PEDOT:PSS film. Additional confirmation of PSS removal from PEDOT:PSS film after benzoic acid treatment is analyzed using XPS spectrum. Figure 4d shows XPS spectra of pristine PEDOT:PSS or PEDOT:PSS with DMSO, methanol, and benzoic acid treatments. While the S2p peak (S1) at $164 \mathrm{eV}$ originates from the thiophene rings of sulfur atoms in PEDOT, the S2p peak (S2) at $168 \mathrm{eV}$ originates from the sulfonate moiety of sulfur atoms in $\mathrm{PSS}^{41,42}$. While the intensity ratio of the $\mathrm{S} 1$ to the S2 (S1/S2) of pristine PEDOT:PSS is 2.1, those of the PEDOT:PSS with DMSO, methanol, and benzoic acid treatment are 1.40,1.35, and 1.09, respectively. The S1/S2 ratio of PEDOT:PSS with benzoic acid treatment is remarkably reduced by $48 \%$ than pristine PEDOT:PSS, indicating of peeling of the broken parts of the PSSH chains on the PEDOT:PSS surface. Figure 4e shows that thickness of the pristine PEDOT:PSS, and PEDOT:PSS with DMSO, methanol, and benzoic acid treatments. The thickness of the pristine PEDOT:PSS, and PEDOT:PSS with DMSO, methanol, and benzoic acid treatments are $63,45,54$, and $38 \mathrm{~nm}$, respectively. The thickness of the PEDOT:PSS films with all treatments are thinner than that of the pristine PEDOT:PSS film. In particular, the thickness of the PEDOT:PSS film after benzoic acid treatment is most reduced from 63 to $38 \mathrm{~nm}$, indicating that the insulating PSSH on the PEDOT:PSS surface can be best removed among various treatment methods. Figure $4 \mathrm{f}$ shows the variations in the ratios of the sheet resistance $\left(R_{0}\right)$ to the initial sheet resistance $\left(R_{i}\right)$ for the pristine PEDOT:PSS electrodes and for the PEDOT:PSS with benzoic acid treatment $(70 \%)$ at $80 \%$ humidity. When the ratios of $\mathrm{R}_{0} / \mathrm{R}_{\mathrm{i}}$ for the pristine PEDOT:PSS electrodes and for the PEDOT:PSS electrodes with benzoic acid treatment are 1/2, their lifetimes are 13.3 and $25.7 \mathrm{~h}$, respectively. Because the PEDOT:PSS electrode absorbs moisture from the atmosphere, the sheet resistances of the pristine PEDOT:PSS films sharply increase over time. However, the sheet resistance of the PEDOT:PSS electrode with benzoic acid treatment slowly increases in comparison with that of the pristine PEDOT:PSS electrode. The stability under humidity of the PEDOT:PSS electrode with benzoic acid treatment is enhanced because the amount of PSS at the surface that absorbs moisture is reduced by the benzoic acid treatment and because the more compact structural rearrangement of the PEDOT:PSS with benzoic acid treatment improves stability under humid conditions ${ }^{43,44}$. The improved stability of the PEDOT:PSS electrode with benzoic acid treatment can increase the lifetime of the OLEDs based on such electrodes.

Figure 5 shows the AFM topography and phase images of (a, e) pristine PEDOT:PSS, and PEDOT:PSS with $(b, f)$ methanol, (c, g) DMSO, and $(\mathrm{d}, \mathrm{h})$ benzoic acid treatments. When the root-mean-square (RMS) roughness of pristine PEDOT:PSS film is $0.88 \mathrm{~nm}$, the RMS roughness of PEDOT:PSS films with methanol, DMSO, and benzoic acid treatment are $0.97,1.08$, and $1.59 \mathrm{~nm}$, respectively. The RMS value of PEDOT:PSS benzoic acid treatment has the largest RMS value of all PEDOT:PSS samples. The insulating PSS shells become separated from the conductive PEDOT chain with increasing screening effect and $\mathrm{H}^{+}$molar \%, thereby increasing the aggregation region of the conductive PEDOT. An increase in the aggregation region of the conductive PEDOT 
(a)

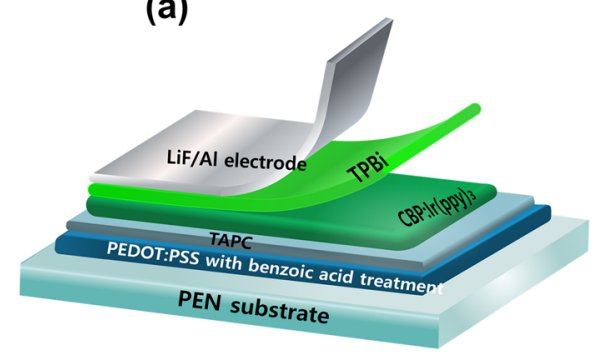

(c)

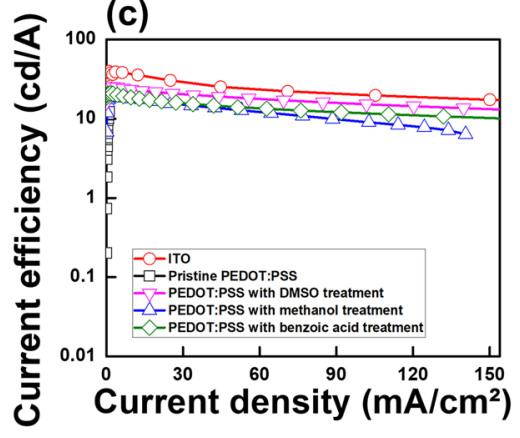

(b)

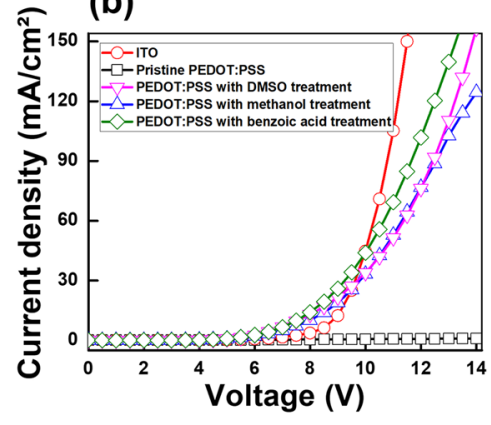

(d)

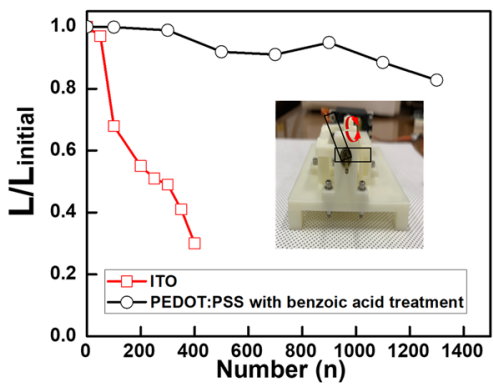

(c)

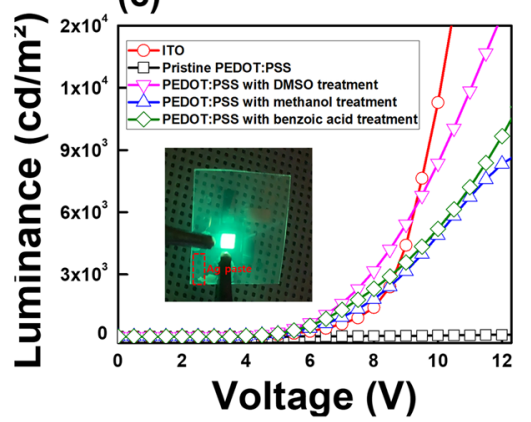

(e)

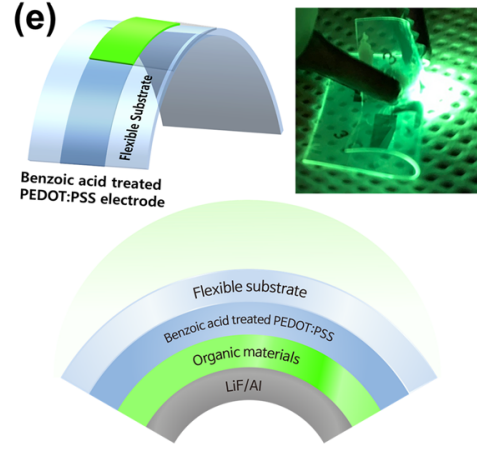

Figure 6. (a) Schematic diagram, (b) current density - voltage, (c) luminance - voltage, (d) current efficiency - current density, (e) bending stability of flexible OLEDs, (f) schematic illustrations of bending test and photographic images of flexible OLED using the PEDOT:PSS electrode with benzoic acid treatment (Kang et al.).

is indicated by an increases in the RMS value, which means an increase in conductivity of PEDOT:PSS film with benzoic acid treatment.

For more reliable analysis, the AFM phase measurement was performed on the PEDOT:PSS films. The associated variation in contrast in the phase images is related to the change in the chemical composition of the PEDOT:PSS film. While the red region can be assigned to a relatively hard phases attributed to the PEDOT rich region in measured phase images under tapping mode, the blue region related to the lower phases indicates the excess of PSS ${ }^{45-47}$. The phase image of the pristine PEDOT:PSS film does not show a distinct change in contrast, as shown in Fig. 5e. This means that the PEDOT chain mixes well with the PSS chain, and the film is mostly covered with PSS rich domain. When the PSSH chains are separated on the surface of the PEDOT:PSS film due to the various post treatments, the shape of the extended PEDOT coil on the surface is reconstructed resulting in the increase of the phase variation ${ }^{48,49}$. The phase variation of the PEDOT:PSS with benzoic acid treatment is more distinct than those of the PEDOT:PSS with methanol and DMSO treatments, as shown in Fig. 5f-h. While the increase of PEDOT rich regions of PEDOT:PSS with methanol and DMSO treatment originates from the screening effect, the increase in PEDOT:PSS with benzoic acid treatment is caused not only by the screening effect, but also by an increase in the amount of $\mathrm{H}^{+}$ions. Therefore, in the benzoic acid treatment, the loss of the PSSH on the PEDOT:PSS increases compared to other post treatment methods resulting in the increase of the conductive PEDOT rich regions.

Figure 6a shows a schematic diagram of the structure of the OLEDs utilizing the PEDOT:PSS with benzoic acid treatment. The device structure is glass substrate/PEDOT:PSS anode with benzoic acid treatment $(38 \mathrm{~nm}) / 1,1$-Bis [(di-4-tolylamino)phenyl] cyclohexane (TAPC) hole-transport layer (HTL) $(40 \mathrm{~nm}) / 4,4^{\prime}$ - $\mathrm{Bis}(\mathrm{N}$ carbazolyl)-1,1'-biphenyl (CBP):Ir(ppy) ${ }_{3}$ emitting layer (EML) $(30 \mathrm{~nm}) / 2,2^{\prime}, 2^{\prime \prime}$ - $(1,3,5$-Benzinetriyl)-tris $(1$ phenyl-1-H-benzimidazole) (TPBi) electron transport layer (ETL) $(30 \mathrm{~nm}) / \mathrm{LiF}$ electron injection layer (EIL) $(1 \mathrm{~nm}) / \mathrm{Al}$ cathode $(100 \mathrm{~nm})$. Figure $6 \mathrm{~b}$ shows the current density-voltage characteristics for OLEDs using ITO, and PEDOT:PSS with DMSO, methanol, and benzoic acid treatments. The OELDs with pristine PEDOT:PSS electrode has a current density of less than $1 \mathrm{~mA} / \mathrm{cm}^{2}$ due to the low conductivity of the pristine PEDOT:PSS electrode. The operating voltages at a current density of $10 \mathrm{~mA} / \mathrm{cm}^{2}$ of the OLEDs using the PEDOT:PSS electrode with DMSO, methanol, and benzoic acid treatments are 7.8, 8.0, and 7.5 V, respectively. The luminance-voltage characteristics for OLEDs using ITO, and PEDOT:PSS with DMSO, methanol, and benzoic acid treatments are shown in Fig. 6c. The pristine PEDOT:PSS electrode could not be operated at luminance up to a luminance of $100 \mathrm{~cd} / \mathrm{m}^{2}$ due to the low conductivity of the PEDOT:PSS electrode. The operating voltages at a luminance of $1000 \mathrm{~cd} / \mathrm{m}^{2}$ of the OLEDs using the PEDOT:PSS electrode with DMSO, methanol, and benzoic acid treatments are 6.7, 7.1, and $5.9 \mathrm{~V}$, respectively. The high conductivity of the PEDOT:PSS electrode with benzoic acid treatment can facilitate the current flow in the OLED, which leads to an enhancement in the current density. Among the post-treatment methods of PEDOT:PSS electrodes, the operating voltage at $1000 \mathrm{~cd} / \mathrm{m}^{2}$ of OLEDs using the PEDOT:PSS electrode with the benzoic acid treatment is the lowest, and its maximum luminance is $24,400 \mathrm{~cd} /$ 
$\mathrm{m}^{2}$, which indicates 1.54 and 2.15 times higher than those of OLEDs using the PEDOT:PSS electrode with the DMSO and methanol treatment, respectively, because of the higher conductivity of PEDOT:PSS electrode with benzoic acid treatment than other post treatments. Figure $6 \mathrm{~d}$ shows the current efficiency-current density characteristics for OLEDs. The current efficiency is related to the charge balance of hole and electron in EML of OLEDs. The maximum current efficiencies of the OLED using PEDOT:PSS electrode with DMSO, methanol, and benzoic acid treatments are 20.9, 18.8 , and $25.3 \mathrm{~cd} / \mathrm{A}$, respectively. The current efficiency of OLEDs using PEDOT:PSS electrode with treated benzoic acid treatments is higher than those of OLEDs using PEDOT:PSS electrode with DMSO and methanol treatments due to the higher conductivity of PEDOT:PSS electrode with treated benzoic acid treatment. The maximum current efficiencies of the OLED using the PEDOT:PSS electrode with benzoic acid treatment and of the OLED with an ITO electrode are 25.3 and $39.8 \mathrm{~cd} / \mathrm{A}$, respectively. The maximum current efficiency of the OLED using the PEDOT:PSS electrode with benzoic acid treatment is smaller than that of the OLED with an ITO electrode due to the high conductivity and the smooth surface morphology of the ITO electrode. However, the PEDOT:PSS electrode is still better than the ITO electrode for use in flexible and transparent OLEDs due to its strength, flexibility, and durability. Figure 6e shows the bending stability of the flexible OLEDs using the ITO electrode and the PEDOT:PSS electrode with benzoic acid treatment at $5 \mathrm{~mm}$ of the bending radius. The luminance of the flexible OLEDs based on ITO electrode is $30 \%$ of the initial luminance value after 400 bending cycles. The surface of the ITO electrode can form the crack after bending due to inherent brittleness of ITO, resulting in a sharply decrease in the luminance of the flexible OLED. On the other hand, the flexible OLED using PEDOT:PSS electrode with benzoic acid treatment is $83 \%$ of the initial luminance after 1400 bending cycles. The schematic illustrations of the bending test for flexible OLEDs, which use the inner bending method, and the photograph of the flexible OLED using PEDOT electrode with benzoic acid treatment after bending are shown in Fig. 6f. When the flexible OLEDs using PEDOT:PSS with benzoic acid treatment is bent to a radius of $5 \mathrm{~mm}$, the flexible OLEDs using PEDOT:PSS with benzoic acid treatment operates well. In the inner bending process, flexible OLEDs might have a small decrease in luminance due to the locally delaminated PEDOT:PSS on the PEN substrate ${ }^{50}$, the increase of sheet resistance is very small, resulting in the excellent bending stability of the PEDOT:PSS electrode with benzoic acid treatment.

\section{Conclusion}

We report on the conductivity enhancement in the PEDOT:PSS electrode with benzoic acid treatment for application in highly efficient, flexible OLEDs. The conductivity of PEDOT:PSS with benzoic acid treatment enhances up to $1538 \mathrm{~S} / \mathrm{cm}$ due to the increases of the high dielectric constant and an amount of the $\mathrm{H}^{+}$ion of the benzoic acid solution. The insulating PSS shells become separated from the conductive PEDOT core with increasing the dielectric constant and $\mathrm{H}^{+}$molar \%, thereby increasing the aggregation region of the conductive PEDOT. While the operating voltages of the OLED using the PEDOT:PSS electrode with benzoic acid treatment at a luminance of $1000 \mathrm{~cd} / \mathrm{m}^{2}$ is $5.9 \mathrm{~V}$, the maximum current efficiency is $25.3 \mathrm{~cd} / \mathrm{A}$, which is much better than OLEDs using PEDOT:PSS electrode with DMSO and methanol treatment. The enhanced performances of OLEDs using the PEDOT:PSS electrode with benzoic acid treatment is attributed to an increase the conductivity of PEDOT:PSS film. The flexible OLED using the PEDOT:PSS electrode with benzoic acid treatment is $83 \%$ of the initial luminance after 1400 bending cycles at a bending radius $5 \mathrm{~mm}$. The PEDOT:PSS electrode with benzoic acid treatment can be used as an alternative high conductive, transparent electrode for flexible OLEDs.

Received: 14 October 2020; Accepted: 27 January 2021

Published online: 16 February 2021

\section{References}

1. Hecht, D. S., Hu, L. \& Irvin, G. Emerging transparent electrodes based on thin films of carbon nanotubes, graphene, and metallic nanostructures. Adv. Mater. 23, 1482-1513 (2011).

2. Ellmer, K. Past achievements and future challenges in the development of optically transparent electrodes. Nat. Photon. 6, 809-817 (2012).

3. Shi, S. W. et al. Solution-processable graphene oxide as an efficient hole injection layer for high luminance organic light-emitting diodes. J. Mater. Chem. C 1, 1708-1712 (2013).

4. Lian, L., Xi, X., Dong, D. \& He, G. Highly conductive silver nanowire transparent electrode by selective welding for organic light emitting diode. Org. Electron. 60, 9-15 (2018).

5. Kou, P. F., Yang, L., Chang, C. \& He, S. Improved flexible transparent conductive electrodes based on silver nanowire networks by a simple sunlight illumination approach. Sci. Rep. 7, 42052 (2017).

6. Liu, Y. A. et al. Capillary-force-induced cold welding in silver-nanowire-based flexible transparent electrodes. Nano Lett. 17, 1090-1096 (2017).

7. Wang, Y. et al. Flexible organic light-emitting devices with copper nanowire composite transparent conductive electrode. J. Mater. Sci. 54, 2343-2350 (2019).

8. Hu, Z. et al. A critical review on semitransparent organic solar cells. Nano Energy 78, 105376 (2020).

9. Hu, Z. et al. Semitransparent ternary nonfullerene polymer solar cells exhibiting $94.0 \%$ efficiency and $24.6 \%$ average visible transmittance. Nano Energy 55, 424-432 (2019).

10. Hu, Z., Wang, Z. \& Zhang, F. Semitransparent polymer solar cells with $9.06 \%$ efficiency and $27.1 \%$ average visible transmittance obtained by employing a smart strategy. J. Mater. Chem. A 7, 7025-7032 (2019).

11. Bae, S. et al. Roll-to-roll production of 30-inch graphene films for transparent electrodes. Nat. Nanotechnol. 5, 574-578 (2010).

12. Feng, L. X. et al. Flexible solid-state supercapacitors with enhanced performance from hierarchically graphene nanocomposite electrodes and ionic liquid incorporated gel polymer electrolyte. Adv. Funct. Mater. 28, 1704463 (2018).

13. Choi, J. Y. et al. Flexible and robust thermoelectric generators based on all-carbon nanotube yarn without metal electrodes. ACS Nano 11, 7608-7614 (2017).

14. Ma, X. et al. Simultaneously improved efficiency and average visible transmittance of semitransparent polymer solar cells with two ultra-narrow bandgap nonfullerene acceptors. J. Mater. Chem. A 6, 21485-21492 (2018). 
15. Chang, Y. M., Wang, L. Y. \& Su, W. F. Polymer solar cells with Poly(3,4-ethylenedioxythiophene) as transparent anode. Org. Electron. 9, 968-973 (2008).

16. Zhao, P. F., Tang, Q. X., Zhao, X. O., Tong, Y. H. \& Liu, Y. C. Highly stable and flexible transparent conductive polymer electrode patterns for large-scale organic transistors. J. Colloid Interface Sci. 520, 58-63 (2018).

17. Kim, D. H., Park, N. H. \& Kim, T. W. Highly efficient flexible organic light-emitting devices based on PEDOT:PSS electrodes doped with highly conductive Pyronin B. Nano Energy 65, 104027 (2019).

18. Kong, B. K., Kim, D. H. \& Kim, T. W. Significant enhancement of out-coupling efficiency for yarn-based organic light-emitting devices with an organic scattering layer. Nano Energy 70, 104503 (2020).

19. Kee, S. Y. et al. Controlling molecular ordering in aqueous conducting polymers using ionic liquids. Adv. Mater. 28, 8625-8631 (2016).

20. Wei, H. Y. et al. Organic solar cells featuring nanobowl structures. Energy Environ. Sci. 6, 1192-1198 (2013).

21. Groenendaal, B. L., Jonas, F., Freitag, D., Pielartzik, H. \& Reynolds, J. R. Poly(3,4-ethylenedioxythiophene) and its derivatives: past, present, and future. Adv. Mater. 12, 481-494 (2000).

22. Zhou, L. et al. Screen-printed Poly(3,4-ethylenedioxythiophene):Poly(styrenesulfonate) grids as ITO-free anodes or flexible organic light-emitting diodes. Adv. Funct. Mater. 28, 1705955 (2018).

23. Alemu, D., Wei, H. Y., Ho, K. C. \& Chu, C. W. Highly conductive PEDOT:PSS electrode by simple film treatment with methanol for ITO-free polymer solar cells. Energy Environ. Sci. 5, 9662-9671 (2012).

24. Ouyang, J. Y., Chu, C. W., Chen, F. C., Xu, Q. F. \& Yang, Y. Polymer optoelectronic devices with high-conductivity Poly (3,4ethylenedioxythiophene) anodes. J. Macromol. Sci. Part A Pure Appl. Chem. 41, 1497-1511 (2004).

25. Liu, S. Y., Deng, H., Zhao, Y., Ren, S. J. \& Fu, Q. The optimization of thermoelectric properties in a PEDOT:PSS thin film through post-treatment. RSC Adv. 5, 1910-1917 (2015).

26. Singh, R. H., Tharion, J. S., Murugan, S. T. \& Kumar, A. ITO-free solution-processed flexible electrochromic devices based on PEDOT:PSS as transparent conducting electrode. ACS Appl. Mater. Interfaces 9, 19427-19435 (2017).

27. Xia, Y. J., Zhang, H. M. \& Ouyang, J. Y. Highly conductive PEDOT:PSS films prepared through a treatment with zwitterions and their application in polymer photovoltaic cells. J. Mater. Chem. 20, 9740-9747 (2010).

28. Xia, Y. J. \& Ouyang, J. Y. Salt-induced charge screening and significant conductivity enhancement of conducting Poly(3,4-ethyle nedioxythiophene:Poly(styrenesulfonate). Macromolecules 42, 4141-4147 (2009).

29. Xia, Y. J. Ouyang, Significant conductivity enhancement of conductive Poly(3,4-ethylenedioxythiophene):Poly(styrenesulfonate) films through a treatment with organic carboxylic acids and inorganic acids. ACS Appl. Mater. Interfaces 2, 474-483 (2010).

30. Kumar, S. R. S., Kurra, N. D. \& Alshareef, H. N. Enhanced high temperature thermoelectric response of sulphuric acid treated conducting polymer thin films. J. Mater. Chem. C 4, 215-221 (2016)

31. Lee, J. H. et al. Highly conductive, stretchable, and transparent PEDOT:PSS electrodes fabricated with triblock copolymer additives and acid treatment. ACS Appl. Mater. Interfaces 10, 28027-28035 (2018).

32. Blessmann, L. et al. Highly conducting, transparent PEDOT:PSS polymer electrodes from post-treatment with weak and strong acids. Adv. Mater. 5, 1800654 (2019).

33. Meng, W. et al. Conductivity enhancement of PEDOT:PSS films via phosphoric acid treatment for flexible all-plastic solar cells. ACS Appl. Mater. Interfaces 7, 14089-14094 (2015).

34. Yu, Z., Xia, Y., Du, D. \& Ouyang, J. PEDOT:PSS films with metallic conductivity through a treatment with common organic solutions of organic salt and their application as a transparent electrodes of polymer solar cells. ACS Appl. Mater. Interfaces $\mathbf{8}$, 11629-11638 (2016).

35. Park, S. Y., Lee, Y. M., Kwak, K. J., Jung, Y. S. \& Kwon, O. H. Alcohol dimer is requisite to form an alkyl oxonium ion in the proton transfer of a strong (photo)acid to alcohol. Chem. Eur. J. 22, 4340-4344 (2016).

36. Kim, J. Y., Jung, J. H., Lee, D. E. \& Joo, J. Enhancement of electrical conductivity of poly(3,4-ethylenedioxythiophene)/poly(4styrenesulfonate) by a change of solvents. Synth. Met. 126, 311-316 (2002).

37. McCarthy, J. E., Hanley, C. A., Brennan, L. J., Lambertini, V. G. \& Gun'ko, Y. K. Fabrication of highly transparent and conducting PEDOT:PSS films using a formic acid treatment. J. Mater. Chem. C 2, 764-770 (2014).

38. Lang, U., Muller, E., Naujoks, N. \& Dual, J. Microscopical investigations of PEDOT:PSS thin films. Adv. Funct. Mater. 19, 1215-1220 (2009).

39. Xia, Y. J. \& Ouyang, J. Y. PEDOT:PSS films with significantly enhanced conductivities induced by preferential solvation with cosolvents and their application in polymer photovoltaic cells. J. Mater. Chem. 21, 4927-4936 (2011).

40. Kim, Y. H. et al. Highly conductive PEDOT:PSS electrode with optimized solvent and thermal post-treatment for ITO-free organic solar cells. Adv. Funct. Mater. 21, 1076-1081 (2011).

41. Crispin, X. et al. Conductivity, morphology, interfacial chemistry, and stability of Poly(3,4-ethylene dioxythiophene)-Poly(styrene sulfonate): a photoelectron spectroscopy study. J. Polym. Sci. Part B Polym. Phys. 41, 2561-2583 (2003).

42. Crispin, X. et al. The origin of the high conductivity of Poly(3,4-ethylenedioxythiophene)-Poly(styrenesulfonate) (PEDOT-PSS) plastic electrodes. Chem. Mater. 18, 4354-4360 (2006).

43. Savagatrup, S. et al. Plasticization of PEDOT:PSS by common additives for mechanically robust organic solar cells and wearable sensors. Adv. Funct. Mater. 25, 427-436 (2014).

44. Nardes, A. M. et al. Conductivity, work function, and environmental stability of PEDOT:PSS thin films treated with sorbitol. $\mathrm{Org}$. Electron. 9, 727-734 (2008).

45. Wang, Y., Song, R., Li, Y. \& Shen, J. Understanding tapping-mode atomic force microscopy data on the surface of soft block copolymers. Surf. Sci. 53, 136-148 (2003).

46. Volkov, A. V. et al. Understanding the capacitance of PEDOT: PSS. Adv. Funct. Mater. 27, 1700329 (2017).

47. Lu, B. et al. Pure PEDOT: PSS hydrogels. Nat. Commun. 10, 1-10 (2019).

48. Sangeeth, C. S. S., Jaiswal, M. \& Menon, R. Correlation of morphology and charge transport in poly(3,4-ethylenedioxy-thiophene)polystyrenesulfonic acid (PEDOT-PSS) films. J. Phys. Condens. Matter. 21, 072101 (2009).

49. Niu, Q. et al. Understanding the mechanism of PEDOT:PSS modification via solvent on the morphology of perovskite films for efficient solar cells. Synth. Met. 243, 17-24 (2018).

50. Cho, C. K. et al. Mechanical flexibility of transparent PEDOT:PSS electrodes prepared by gravure printing for flexible organic solar cells. Sol. Energy Mater. Sol. Cells 95, 3269-3275 (2011).

\section{Acknowledgments}

This research was supported by the Basic Science Research Program through the National Research Foundation of Korea (NRF) funded by the Ministry of Education, Science and Technology (2019R1A2B5B03069968).

\section{Author contributions}

T.W.K. conceived the project, and H.S.K. and D.H.K. designed and performed the experiments and collected the data. H.S.K. and D.H.K. analyzed and discussed the data. All authors discussed the results and contributed to the writing of the manuscript. 


\section{Competing interests}

The authors declare no competing interests.

\section{Additional information}

Correspondence and requests for materials should be addressed to T.W.K.

Reprints and permissions information is available at www.nature.com/reprints.

Publisher's note Springer Nature remains neutral with regard to jurisdictional claims in published maps and institutional affiliations.

(c) (i) Open Access This article is licensed under a Creative Commons Attribution 4.0 International License, which permits use, sharing, adaptation, distribution and reproduction in any medium or format, as long as you give appropriate credit to the original author(s) and the source, provide a link to the Creative Commons licence, and indicate if changes were made. The images or other third party material in this article are included in the article's Creative Commons licence, unless indicated otherwise in a credit line to the material. If material is not included in the article's Creative Commons licence and your intended use is not permitted by statutory regulation or exceeds the permitted use, you will need to obtain permission directly from the copyright holder. To view a copy of this licence, visit http://creativecommons.org/licenses/by/4.0/.

(c) The Author(s) 2021 